Fisioter Bras 2017;18(3):257-9

\title{
EDITORIAL \\ Cuidados paliativos na esclerose lateral amiotrófica
}
Marco Orsini ${ }^{*}$, Viviane Marques ${ }^{* *}$, Carlos Henrique Melo Reis ${ }^{* * *}$, Mauricio de Sant Anna $\mathrm{Jr}^{* * * *}$, Adriana Leico Oda ${ }^{* * * *}$, Victor Hugo Bastos ${ }^{* * * * *}$, Silmar Teixeira ${ }^{* * * * *}$, Marcos RG de Freitas $^{* \star *}$, Acary Bulle Oliveira ${ }^{* \star * *}$

*Universidade Severino Sombra, Programa de Mestrado em Ciências Aplicadas à Saúde e Centro Universitário Augusto Motta, Ciências da Reabilitação, Rio de Janeiro, **Universidade Federal do Estado do Rio de Janeiro, UNIRIO, Departamento de Fonoaudiologia e

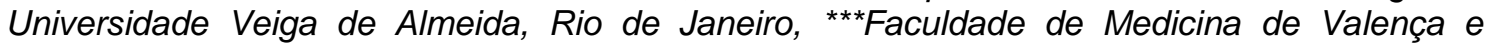
Serviço de Neurologia do Hospital Geral de Nova Iguaçu, Rio de Janeiro, ${ }^{* * *}$ Instituto Federal do Rio de Janeiro, IFRJ, Curso de Fisioterapia, ${ }^{\star * * \star *}$ Escola Paulista de Medicina, Universidade Federal de São Paulo, UNIFESP, ${ }^{* * * * * U n i v e r s i d a d e ~ F e d e r a l ~ d o ~ P i a u i, ~ D e p a r t a m e n t o ~ d e ~}$ Medicina

Endereço para correspondência: Marco Orsini, Rua Professor Miguel Couto, 322/1001, Jardim Icaraí 24230240 Niterói RJ, E-mail: orsinimarco@hotmail.com; Viviane Marques: vivianemarquesfono@hotmail.com; Carlos Henrique Melo Reis: chmeloreis@hotmail.com; Mauricio de Sant Anna Jr: fisioenf@gmail.com; Adriana Leico Oda: adrileico.oda@uol.com.br ; Victor Hugo Bastos: victorhugobastos@ufpi.edu.br; Silmar Teixeira: silmarteixeira@ufpi.edu.br; Marcos RG de Freitas: mgdefreita@hotmail.com; Acary Bulle Oliveira: acary.bulle@unifesp.br

A Esclerose Lateral Amiotrófica (ELA) pode ser definida como uma doença neurológica, progressiva e degenerativa que cursa com o comprometimento dos neurônios da ponta anterior da medula espinhal e do feixe piramidal em graus variados [1]. Os pacientes apresentam deficiências e/ou incapacidades em suas atividades básicas e instrumentais da vida diária, caracterizadas por padrões heterogêneos de deterioração como, por exemplo, dificuldades de locomoção, comunicação, respiração, deglutição, além de mudanças de humor e comportamento. Problemas psicológicos como depressão e ansiedade, ligados à vivência da doença também são comumente relatados [1,2]. Ainda com prognóstico reservado, leva a óbito cerca de 5-8 anos após o início dos sintomas [1].

Acostados estão os princípios da beneficência aos padecentes de suas condições saúde inexoráveis, tanto nos textos sagrados, quanto nos ensinamentos de Hipócrates, patrono da medicina ocidental. Desta feita, os cuidados paliativos na ELA deveriam ser considerados já no início da trajetória do adoecimento, tão somente no aguardo do tempo certo de sua instituição, ou seja, devem fazer parte do elenco de estratégias de tratamento instituído por parte do neurologista, sem prejuízos das intervenções neurológicas propostas, com o intuito de oportunizar a ação de equipe multiprofissional, que inclui a assistência integral do paciente e de seus familiares. Na ausência de tratamento curativo, os cuidados podem melhorar a qualidade de vida, prolongar a sobrevivência e apoiar os portadores de ELA e suas famílias e, principalmente, ajudá-los a antecipar e se preparar para o fim da vida [3].

$\mathrm{O}$ atendimento domiciliar adequado associado às medidas de paliação mostra-se mais vantajoso que internações desnecessárias. A atenção domiciliar e os cuidados paliativos (CP) na ELA remontam aos princípios da humanidade no cuidado, não substituindo a assistência hospitalar, mas sendo uma proposta de tratamento que possibilita ao paciente a manutenção da sua autonomia, visto que os cuidados no domicílio são executados no tempo do paciente e do seu núcleo familiar. Uma questão de significativa relevância é o menor risco de exposição dos pacientes a agentes infecciosos, reduzindo à associação de co-morbidades nosocomiais [3].

No tocante a pacientes com ELA algumas vantagens do serviço de atendimento domiciliar multidisciplinar devem ser destacadas: desospitalização precoce; redução de custos; treinamento de familiares com programas que facilitam a comunicação alternativa não-verbal; gerenciamento da oferta via oral total, parcial ou suspensão da alimentação realizando manutenção do prazer oral; possibilidade de uma equipe interdisciplinar treinada para monitorar a condição clínica dos pacientes; conforto respiratório com adequação dos padrões 
ventilatórios; acompanhamento do status nutricional e metabólico. O envolvimento das clínicas especializadas compostas por médicos, enfermeiros, fisioterapeutas, fonoaudiólogos, psicólogos, nutricionistas promovem ações programadas e continuadas que podem ampliar a sobrevivência e melhorar o conforto no fim de vida [4,5].

Entendamos por $\mathrm{CP}$, práticas voltadas para o período final da vida de pacientes fora de condutas terapêuticas de cura, que reflete a mudança de paradigma e de conceitos sobre a matéria do corpo, o adoecimento e a morte. Tais práticas buscam em última análise, o controle dos sinais e sintomas físicos e psicológicos próprios do estágio avançado da ELA, além da melhora da qualidade de vida [6]. Os CP se desenvolveram em grande parte devido aos "excessos" cometidos pela medicina, que conduzia, até pouco tempo, o paciente a um final de vida medicalizado e carregado de sofrimento, em contraposição à qualidade do viver [6,7]. Acreditamos que na ELA os cuidados paliativos devem estar de prontidão desde 0 estabelecimento do diagnóstico de certeza até o estágio realmente terminal da doença.

A reabilitação também assume a responsabilidade tanto dos cuidados respiratórios como músculo-esqueléticos dos pacientes portadores de ELA. Esses cuidados passam pela utilização de exercícios ativos (quando possível) e passivos, que visam à adequada mobilização do paciente impedindo o surgimento não só de deformidades e encurtamentos, mas como o surgimento de úlceras por pressão. No que tange aos cuidados respiratórios, a monitorização constante da função dos músculos ventilatórios, saturação de oxigênio tanto diurna quanto noturna, assim como o pico de fluxo torna-se primordiais, principalmente no tocante a definição da instituição tanto do suporte ventilatório não invasivo, e posteriormente traqueostomia [8].

A manutenção da permeabilidade das vias aéreas, através de adequada higienização é uma atividade que deve ser realizada não só pelo fisioterapeuta, mas por todos os profissionais da saúde. Essas estratégias minimizam a ocorrência de pneumonias broncoaspirativas [9].

O fim da vida implica dilemas éticos e impasses de natureza jurídica, fazendo com que todos os envolvidos nesse cenário reflitam de forma crítica a respeito da conduta bioética e judicial mais adequada, ante a terminalidade da vida humana. Trata-se, pois, de um desafio real aos serviços e equipes de saúde, que precisam ser enfrentados [8].

Com os conhecimentos contemporâneos, não há sustentação para que os pacientes com ELA permaneçam internados longamente, porquanto existe um dever moral e ético em fornecer cuidados domiciliares. Devido a complexidade e especificidade dos aspectos clínicos e do espectro de apresentação da ELA, os profissionais devem ser exaustivamente treinados $[1,7,9]$. Nossa expectativa para o atendimento domiciliar de pacientes em estágio terminal de ELA é otimista. Logicamente, são necessários aprimoramentos nos cuidados específicos. Cabe-nos lutar para garantir tal oportunidade.

Referências

1. Hogden A et al. Amyotrophic lateral sclerosis: improving care with a multidisciplinary approach. J Multidiscip Healthc 2017;10:205.

2. Caga J, Ramsey E, Hogden A, Mioshi E, Kiernan MC. A longer diagnostic interval is a risk for depression in amyotrophic lateral sclerosis. Palliat Support Care 2015;13(4):1019-24.

3. Soriani $\mathrm{M}-\mathrm{H}$, Desnuelle $\mathrm{C}$. Care management in amyotrophic lateral sclerosis. Rev Neurol 2017;173(5):288-99.

4. Traynor BJ, Alexander M, Corr B, Frost E, Hardiman O. Effect of a multidisciplinary amyotrophic lateral sclerosis (ALS) clinic on ALS survival: a population based study. $J$ Neurol Neurosurg Psychiatry 2003;74:1258-61.

5. Oliver DJ et al. A consensus review on the development of palliative care for patients with chronic and progressive neurological disease. Eur J Neurol 2016;23(1):30-8.

6. Menezes RA. Em busca da boa morte: antropologia dos cuidados paliativos. Rio de Janeiro: Garamond/Fiocruz; 2004.

7. Kruse MHL, Vieira RW, Ambrosini L, Niemeyer F, Silva FP. Cuidados paliativos: uma experiência. Rev HCPA 2007;27(2):49-52.

8. Bastos $\mathrm{VH}$ et al. Therapeutic exercise in amyotrophic lateral sclerosis: what do we expect from anabolism versus catabolism? EC Neurology 2017;6(1): 24-5.

9. Orsini M. Amyotrophic Lateral Sclerosis (ALS) and physical rehabilitation: double standards. J J Physical Rehab Med 2016:2(4): 027. 
10. Brito FM et al. Cuidados paliativos e comunicação: estudo com profissionais de saúde do serviço de atenção domiciliar Palliative care and communication: study with health professionals of the home care service. Revista de Pesquisa: Cuidado é Fundamental Online 2017;9(1):215-21.

11. Caraceni A, de Viner M, Grisold W et al. Palliative care in chronic and progression neurological disease: summary of a consensus review. European Journal of Palliative Care 2016;23(5):232-5. 